\title{
Evaluación Clínica y radiográfica de injertos biocerámicos tipo Hidroxiapatita como alternativa en la reconstrucción de alveolos dentarios postexodoncia
}

\author{
Clinical and radiographic evaluation of grafts biocerámicos type \\ Hidroxiapatita like alternative in the reconstruction of alveoli toothworts \\ postexodoncia
}

\author{
Julián Gallón Nausa
}

\section{Resumen}

Objetivo: Evaluar el comportamiento de la cresta ósea marginal con la plataforma del implante como referencia, en casos de pacientes con injerto aloplástico de la casa farmacéutica Madrob con el fin de mantener la integridad ósea como elemento fundamental en la colocación de implantes dentales. Materiales y métodos: Se llevó a cabo un estudio de tipo comparativo y prospectivo, con una muestra intencional de 30 pacientes para un total de 81 implantes con previo requisito de injerto aloplásticos en alveolos pos exodoncia y posterior implante dental. Se realizaron controles clínicos y radiográficos a los 3, 6, 9, 12, 24 y 36 meses para evaluar el comportamiento de la cresta ósea marginal. Resultados: Hay mayor aumento de pérdida ósea por mesial que por distal entre los 12 y 36 meses. Entre 24 y 36 meses, el aumento es igual por mesial que por distal y es de solo $0,05 \mathrm{~mm}$. Las diferencias entre tiempos son todas significativas $\mathrm{p}<0,001$. El ANOVA indica que todas las varianzas son homogéneas y pequeñas entre $0,18 \mathrm{~mm}$ y $0,22 \mathrm{~mm}$.

Palabras clave: hidroxiapatita, osteoconductivo, integridad ósea, injerto, biocerámica.

\section{Resumen}

Objective: The aim of this work is to evaluate the behavior of the bony marginal comb with the platform of the implant as reference, in patients' cases with graft aloplástico of the pharmaceutical house Madrob in order to support the bony integrity as fundamental element in the placement of dental implants. Materials and methods: There was carried out a study of comparative and market type, with an intentional sample of 30 patients for a total of 81 implants with previous requirement of graft aloplásticos in alveoli pos exodoncia and later dental implant. There were realized clinical and radiographic controls for 3, 6, 9, 12, 24 and 36 months to evaluate the behavior of the bony marginal comb. Results: There is major increase of bony loss for mesial that for distal between 12 and 36 months. Between 24 and 36 months, the increase is equal for mesial that for distal and it is of only $0,05 \mathrm{~mm}$. The differences between times are significant all $\mathrm{p}<0,001$. The ANOVA indicates that all the variances are homogeneous and small between $0,18 \mathrm{~mm}$ and $0,22 \mathrm{~mm}$.

Key words: hydroxyapatite, osteoconductive, bone integrity, graft, bioceramics.

${ }^{10}$ D.,Esp. Cirugía Oral, Magister en Ciencias Pontificia Universidad Javeriana, Docente Hospital San Blas servicio Maxilo Facial. Correspondencia: julianfgallon@gmail.com 


\section{Introducción}

El tejido óseo contiene 33\% de matriz orgánica, la cual incluye un $28 \%$ de colágeno tipo I y $5 \%$ de proteína no colágena (osteonectina, osteocalcina, proteína morfogénetica de hueso, proteoglicano óseo y sialoproteina ósea). La matriz orgánica está impregnada por una hidroxiapatita pobremente cristalizada y deficitaria en calcio que constituye el $67 \%$ restante. Además de sus funciones relacionadas con el esqueleto de soporte, protección y reservorio de minerales, este tejido se encuentra regulado por factores hormonales, localmente por fuerzas mecánicas (incluyendo el movimiento de los dientes) y por condiciones de tipo piezoeléctrico. Aunque las cifras posteriormente indicadas sobre la composición del hueso son aproximadas, el cociente entre los componentes duros y blandos es suficiente como para asegurar un cierto grado de elasticidad. El hueso resiste a fuerzas compresivas mucho mejor que a las fuerzas tensionales. El hueso también resiste a las fuerzas aplicadas a lo largo del eje de su componente fibroso, las fracturas óseas ocurren más fácilmente como respuesta a tensiones y cortes (1-17).

La regeneración de defectos óseos por causa de traumas directos como fracturas, secuelas oncológicas o simplemente la pérdida de la altura ósea, durante décadas se ha convertido en un reto buscando en los injertos ciertas características que respondan a cada una de las necesidades de reconstrucción del macizo facial. El material sustitutivo del hueso debe cumplir con los postulados emitidos por Scales 1953 y Ashley 1967 donde se estima que los injertos no deben ser modificados por los tejidos blandos, no pueden causar reacciones inflamatorias, ser esterilizables, no sufrir desplazamientos, ni tener características carcinógenas.

De esta forma, se presenta como alternativa a este tipo de injertos las cerámicas tipo fosfatos de calcio como la hidroxiapatita (HA) sintética biocerámica, con capacidad de unirse químicamente con el tejido óseo haciéndola bioactiva, entre sus propiedades se incluye: cristalinidad, pureza, densidad, morfología, porosidad y microestructura, estas características fisicoquímicas y mecánicas con el tejido óseo hacen que se comporte como una estructura osteoconductiva que permite ser invadida por tejido conectivo proveniente del hueso de la periferia para posteriormente osificarse, manteniendo las características macroporosas de su origen. Su similitud con el hueso ha permitido que se convierta en una alternativa como sustituto óseo en diferentes tratamientos en cirugía oral y maxilofacial.

La HA sintética, con fines biomédicos de la casa Madrob Farmacéutica, presenta una composición de HA (calcio fosfato tribásico USP, colágeno y proteína morfogenética ósea de porcino BMP 2). Está indicada como material de relleno óseo con factor osteoconductivo. Es importante resaltar la presencia del colágeno el cuál sirve de molde para dar lugar al nuevo cristal que crecerá y se diferenciará para establecerse en las regiones más externas, las de mayor fuerza, mediante un proceso de remodelación.

Por último, se debe resaltar la importancia de las proteínas morfogenéticas óseas péptidos producidos localmente por los osteoblastos, regulados por mecanismos endocrinos. Se encuentran distribuidos a lo largo de las fibras colágenas del hueso normal, en células periostales y mesenquimales de la médula ósea, mediando los eventos celulares con capacidad de adherirse a los receptores superficiales de células blancas. Esta interacción dispara una serie de eventos celulares que pueden influenciar los diferentes procesos de proliferación y diferenciación celular, síntesis de proteínas y producción de matriz extra celular.

\section{Materiales y métodos Población accesible y muestra}

Se llevó a cabo un estudio de tipo observacional comparativo y prospectivo con una muestra intencional de 30 pacientes con un total de 81 
implantes. Como criterios de inclusión, requisito previo de injerto aloplásticos en alveolos pos exodoncia y posterior implante dental. Y de exclusión pacientes con antecedentes clínicos de patologías óseas que puedan afectar el metabolismo óseo.

\section{Exodoncia}

Se tuvo en cuenta para las exodoncia una dosis previa antibiótica de 2 gramos de amoxicilina diarios un día antes y durante 4 días más para conseguir dosis plasmáticas adecuadas en el coágulo primario. Previa asepsia y antisepsia del campo operatorio, se realizó exodoncia, se decorticaron las paredes alveolares y a continuación se perforó el alveolo dental para inducir la formación de un coagulo medular. Posteriormente se hidrató el material aloplástico con solución salina fisiológica, compactando el material y dejando fluir el coágulo medular. No se sobre corrigió el defecto para permitir confrontar los tejidos, los cuales posicionaron los injertos de tejido conectivo tomados de la región anatómica del paladar, con el objetivo de confrontar los tejidos con menor fuerza tensil de las suturas y obtener un mejor selle primario.

\section{Evaluación}

Se realizaron controles clínicos y radiográficos a los 3, 6, 9, 12 ,24 y 36 meses. Primero se evaluó a los 3 y a los 6 meses la integridad del injerto tipo aloplásticos, posteriormente a los 6 meses se colocaron los implantes dentales, y se evaluaron durante los meses restantes controlando la restauración protésica desde los 12, 24 y 36 meses. En los controles clínicos se tuvo en cuenta, como criterio mayor, el no presentar dehiscencias ni signos de infección pos quirúrgica.

El método de evaluación se realizó con radiografías peri apicales con películas de fosforo marca Soderex Imagin Plates, con técnica de paralelismo por medio de aditamentos de la casa Dentsplay. La dosis estimada por placa fue de $7,4 \mu \mathrm{Sv}$ donde se evaluó el comportamiento posicional de la cresta ósea marginal mesial y distal teniendo como referencia la plataforma del implante. Las mediciones fueron tomadas con un calibrador Vernier digital serie 799 con una capacidad de $0-150 \mathrm{~mm}$ y una resolución de $0.01 \mathrm{~mm}$; se realizaron calibraciones cada 10 mediciones. Los datos se organizaron en tablas que relacionan longitud del implante, altura de la cresta mesial y distal, tipo de maxilar y datos demográficos de sexo y edad, adicionalmente, se relacionan con criterios de éxito, supervivencia, mal pronóstico y fracaso de acuerdo con lo postulado por Roos y colaboradores 1997 teniendo como referencia las variables de altura mesial y distal de la cresta ósea

\section{Resultados}

Este estudio pretendió evaluar el comportamiento de injertos aloplásticos en alveolos pos exodoncia y la consiguiente colocación de implantes dentales para estimar la ubicación dimensional de la cresta alveolar mesial y distal, teniendo como referente la plataforma del implante que presenta una superficie maquinada y modificada. Para esto se realizaron controles clínicos y radiográficos a los 3, 6, 12,24 y 36 meses en un total 81 implantes; en la Tabla 1 se presentan los datos demográficos de la muestra de pacientes, distribución por género, que indica mayoría de mujeres en la muestra $57 \%$ y un rango de edad entre los 28 y 67 ańos (promedio 46.4 años) para un total de 30 pacientes.

La cantidad de implantes por paciente es en promedio 2,6, con un rango 1 a 7 implantes, presentando un porcentaje de éxito del $90 \%$ para un total de 60 implantes distribuidos en el maxilar superior $(74 \%)$ y 21 implantes en el maxilar inferior (26\%). De los 81 implantes, 3 fueron catalogados como fracasos. Tabla 1. 
Tabla 1. Datos demográficos de la muestra de pacientes.

\begin{tabular}{|c|c|c|c|c|}
\hline \multicolumn{2}{|c|}{} & Hombres & Mujeres & TOTAL \\
\hline \multirow{2}{*}{$\begin{array}{c}\text { Distribución de la muestra } \\
\text { por géneros }\end{array}$} & $13(43 \%)$ & $17(57 \%)$ & \multirow{2}{*}{30} \\
\hline \multirow{2}{*}{ Edad (años) } & Promedio & Desv.st. & Rango & \\
\cline { 2 - 4 } & 46,4 & 11,3 & 28 a 67 & \\
\hline
\end{tabular}

\begin{tabular}{|l|c|c|}
\cline { 2 - 3 } \multicolumn{1}{c|}{} & Promedio & Rango \\
\hline Cantidad de implantes/pte & 2,6 & 1 a 7 \\
\hline Implantes \% de éxito & \multicolumn{2}{c|}{$90 \%$ (3 fracasos) } \\
\hline Implantes Maxilares & $60(74 \%)$ \\
\hline Implantes Mandibulares & $21(26 \%)$ \\
\hline Total de implantes & \multicolumn{2}{|c|}{$\mathbf{8 1}$} \\
\hline
\end{tabular}

En la Tabla 2 se presentan los resultados de altura del hueso crestal. Cada periodo de tiempo está relacionado de mesial a distal, donde a los 12 meses la cresta mesial presenta un promedio de 0,914 $\pm 0,213$

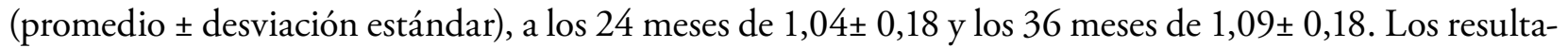
dos de la cresta distal presentaron a los 12 meses un promedio de $1 \pm 0,22$, a los 24 meses de $1,059 \pm 0,18$ y a los 36 meses de $1,109 \pm 0,188$.

Tabla 2. Resultados de altura del hueso crestal en mm, para cada periodo de tiempo y cada superficie.

\begin{tabular}{|c|c|c|c|}
\hline & 12 meses & 24 meses & 36 meses \\
\hline Mesial & $0,914 \pm 0,213$ & $1,04 \pm 0,18$ & $1,09 \pm 0,18$ \\
\hline Distal & $1 \pm 0,22$ & $1,059 \pm 0,18$ & $1,109 \pm 0,188$ \\
\hline
\end{tabular}

La Tabla 3 presenta los resultados del análisis estadístico expresando la probabilidad de la prueba $t$ pareada, que indica que todas las diferencias fueron significativas para un nivel de significación $\mathrm{p}<$ 0,001. Se muestra también la diferencia entre cada periodo tanto en $\mathrm{mm}$ como en porcentaje de cambio respecto al periodo de tiempo anterior.

Tabla 3. Presenta los resultados expresando la probabilidad de la prueba t pareada que indica que todas las diferencias fueron significativas pea un $\mathrm{mm}$ como en porcentaje de cambio respecto al periodo de tiempo anterior.

\begin{tabular}{|c|c|c|c|}
\cline { 2 - 4 } \multicolumn{1}{c|}{} & $\mathbf{p}$ & Aumento (mm) & \% de cambio \\
\hline mesial 12 vs 24 meses & $1,00 \mathrm{E}-09$ & 0,126 & 13,78 \\
\hline mesial 12 vs 36 meses & $5,00 \mathrm{E}-11$ & 0,176 & 19,25 \\
\hline mesial 24 vs 36 meses & 0,00066 & 0,05 & 4,8 \\
\hline distal 12 vs 24 meses & 0,0007 & 0,059 & 5,9 \\
\hline distal 12 vs 36 meses & $3,00 \mathrm{E}-06$ & 0,109 & 10,9 \\
\hline distal 24 vs 36 meses & 0,00026 & 0,05 & 4,7 \\
\hline
\end{tabular}


En la Tabla 4 se relacionan los datos con criterios de éxito, supervivencia, mal pronóstico y fracaso, estimándose como criterios mayores.

Tabla 4. Relación de datos con criterios de éxito, supervivencia, mal pronóstico y fracaso, estimándose como criterios mayores.

\begin{tabular}{|l|c|}
\hline ÉXITO & 78 \\
\hline Supervivientes & 0 \\
\hline Mal pronóstico & 1 \\
\hline Fracasos & 2 \\
\hline
\end{tabular}

En conclusión, hay mayor aumento de pérdida ósea por mesial que por distal entre los 12 y 36 meses. Entre 24 y 36 meses el aumento es igual por mesial que por distal y es de solo $0,05 \mathrm{~mm}$.

Las diferencias entre tiempos son todas significativas $\mathrm{p}<0,001$. El ANOVA indica que todas las varianzas son homogéneas y pequeñas entre 0,18 $\mathrm{mm}$ y $0,22 \mathrm{~mm}$. Figura 3. Los datos siguen una distribución normal y la muestra es de tamaño aceptable $(\mathrm{n}=27)$.
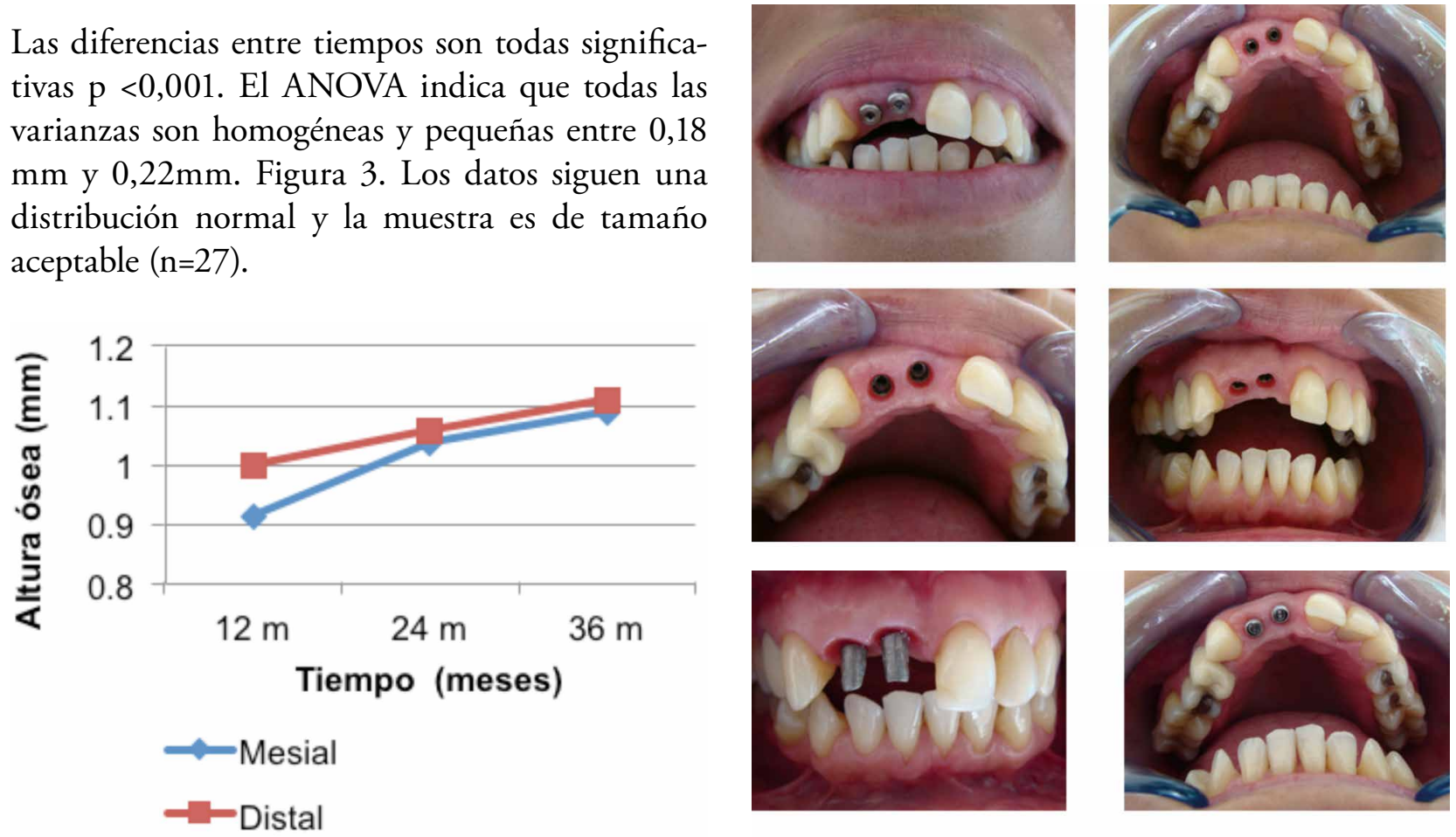

Figura 1. Cambios en la altura ósea crestal, mesial y distal respecto al tiempo.

Resultados del control radiográfico para evaluar el comportamiento de la cresta ósea marginal de 3 meses a 36 meses, así como el control clínico de la fase final de rehabilitación oral se presentan en las Figuras 2 y 3 .
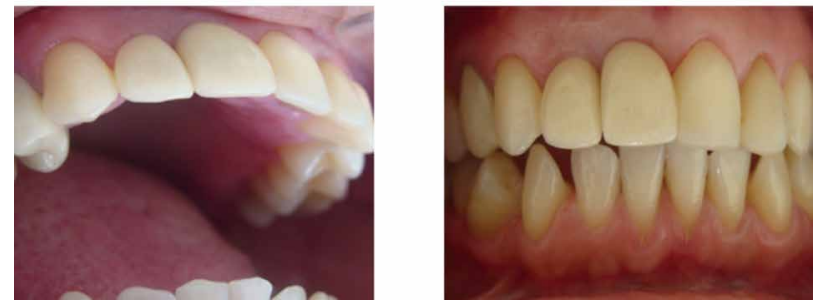

Figura 3. Control clínico, fase final de rehabilitación oral. 


\section{Discusión}

Bajo las condiciones de protocolos de colocación de implantes dentales (16), es importante tener en cuenta parámetros clínicos que evalúan el comportamiento radiográfico de la cresta ósea después de la colocación de implantes osteointegrados, todo esto representa una serie de eventos que se inician desde el mismo diagnóstico clínico, características sistémicas del paciente, seguimiento de protocolos clínicos, acto quirúrgico, compatibilidad de materiales, microambiente celular, propiedades mecánicas y tiempos adecuados de integración biológica; estas variables pueden representar el éxito o fracaso en la colocación de implantes dentales.

Basados en estos parámetros, varios autores han propuesto un límite de $1,5 \mathrm{~mm}$ de pérdida ósea alrededor de implantes osteointegrados exitosamente durante el primer año de carga y un límite para los siguientes ańos de $0,2 \mathrm{~mm}(3,5-16)$. Recientemente el consenso del Congreso Internacional de Implantólogos orales aprobó cuatro categorías clínicas que definen las condiciones de éxito, supervivencia y fracaso de los implantes dentales, en dicho consenso, se estableció que un implante se clasifica en grupo de éxito cuando cumple las siguientes condiciones: ausencia de dolor a la palpación, percusión o función, sin movilidad clínica en cualquier dirección, ausencia de exudado y pérdida ósea marginal radiográfica menor de $2 \mathrm{~mm}$.

Los implantes evaluados en este estudio y su directa relación con la cresta ósea se pueden catalogar en el grupo de éxito a los 12 meses. Los implantes con carga funcional presentaron una pérdida ósea promedio de $0,914 \mathrm{~mm}$ por mesial y $1 \mathrm{~mm}$ por distal. En los siguientes meses 24 y 36 la perdida promedio de la altura de la cresta ósea fue de $1 \mathrm{~mm}$ mostrando una marcada estabilización de la anatomía peri implantar. Arismendi JA, en su estudio comparativo de implantes de superficie lisa y rugosa, resultado a 36 meses, determinó que los implantes de superficie lisa con carga funcional presentaron una pérdida ósea de $0,4 \mathrm{~mm}$, mientras que a los 24 y 36 meses la pérdida ósea fue de $1,5 \mathrm{~mm}$. Igualmente los implantes de superficie rugosa con carga funcional a los 12 meses, demostraron pérdida ósea promedio de $0,6 \mathrm{~mm}$. A los 24 meses dicha pérdida incremento en promedio 1,4 $\mathrm{mm}$, y a los 36 meses se aumentó ligeramente a 1,5 $\mathrm{mm}$ en promedio.

Chang M, Wennstrom JL (9-10) estudiaron 18 pacientes con un total 43 implantes sin ningún tipo de injerto, evaluando las alteraciones del hueso crestal y su relación con tejidos blandos durante 3 años. En los 6 meses después de la cirugía de colocación de implantes de una etapa, el margen del tejido blando retrocedió aproximadamente $0,6 \mathrm{~mm}$ y $1,1 \mathrm{~mm}$ sin reportes significativos, durante los meses siguientes estos mismos autores $(11,12)$ reportan un estudio evaluado en 5 años con controles radiográficos analizando la topografía del hueso proximal en unidades de diente implante con un $\mathrm{n}=36$ comparados con implante implante con un $\mathrm{n}=67$ y un $\mathrm{p}<0,052$. Los resultados reportados demuestran que en las unidades de diente implante la pérdida de hueso proximal era de $0,4 \mathrm{~mm}$ y en las unidades de implante implante es de $0,5 \mathrm{~mm}$, demostrando una mayor pérdida ósea en el espacio de interimplantación. En el presente estudio se evaluaron 81 implantes con requisito previo de exodoncia, injerto aloplástico e implante evaluados durante 3 años donde entre 24 y 36 meses la pérdida es igual por mesial que por distal y es de solo $0,05 \mathrm{~mm}$. Las diferencias entre los tiempos son todas significativas con $\mathrm{p}<0,001$ y comparadas entre unidades de diente implante es de $0.1 \mathrm{~mm}$ y en implante es de $0,6 \mathrm{~mm}$, demostrando como se disminuye la pérdida ósea con injertos previos que asegura una mejor orientación del implante sin carga excesiva a una cortical.

Lars Schropp (3) realizó un estudio comparando los cambios del hueso crestal después de implantes pos-exodoncia contra implantes de 3 meses después de la exodoncia, en 46 pacientes relacionados en dos grupos $n=23$ realizando controles clínicos y radiográficos. Los resultados demostraron una supervivencia del $91 \%$ en la implantación inme- 
diata con una pérdida ósea de $2 \mathrm{~mm}$, un $96 \%$ en el grupo de 3 meses y una pérdida ósea de $1,5 \mathrm{~mm}$ en promedio. En el presente estudio se presentó éxito del $90 \%$ con una altura promedio de la cresta ósea de $1 \mathrm{~mm}$ con varianzas homogéneas y pequeñas entre 0,18 y $0,22 \mathrm{~mm}$ demostrado como injertos previos no solamente son rellenos de cavidades, sino elementos osteoconductivos, que asociados a implantes colocados infracrestalmente, promueven una nueva formación de hueso crestal.

Los resultados sobre pérdida ósea ocurrida en 13 estudios que evalúan el comportamiento de implantes del sistema Branemark, después del primer año de carga funcional, muestran que la disminución ósea se encuentra entre 0,4 y $1,6 \mathrm{~mm}$ con promedio de $0,93 \mathrm{~mm}$. Los resultados obtenidos en esta investigación se encuentran en un promedio de $0,18 \mathrm{~mm}$ con una pérdida ósea de $1 \mathrm{~mm}$ donde se indica que todas las diferencias fueron significativas para un nivel de significación $\mathrm{p}<0,001$.

El límite de adaptación biomecánica del tejido óseo marginal (8), está determinado por una gran cantidad de variables, una de ellas es la superficie de los implantes. Bajo estas condiciones, los nuevos diseños de superficie están encaminados en proporcionar una unidad de contacto biológicamente más funcional que permita una adecuada comunicación intercelular capaz de soportar fuerzas de tipo funcional de una manera eficiente.

Por otra parte, Young (2) estudió las propiedades de nanotubos sobre superficies micro estructuradas de titanio en fémur de conejos, demostrando considerables aumentos en la óseo integración. Revisiones posteriores (1,2-6,13-14) sobre nanoestructuras de superficies de implantes, correlacionan la función estructural de las superficies implantares y el hueso nativo prestando gran importancia a la adherencia de los osteoblastos a las superficies de los implantes (7). En este estudio se utilizaron implantes con superficies tratadas, las cuales proporcionan unidades amplias de contacto celular favoreciendo la oseointegración y mejorando el límite de adaptación biomecánica que desde un punto clínico y radiográfico, están enmarcados en la pérdida ósea crestal y su directa relación con los tejidos blandos.

\section{Conclusión}

El biomaterial evaluado de composición hidroxiapatita, colágeno y proteína morfogenéticas porcina demostró un comportamiento satisfactorio como material de relleno óseo y contribuye a la conservación de la masa ósea en los defectos tratados, lográndose integridad y estabilidad de los implantes estudiados; sin presentar reacciones adversas en ninguno de los casos estudiados.

Como consenso final los parámetros que influyen en el éxito final en cuanto a la remodelación ósea y la formación de hueso nuevo son los siguientes:

- Condiciones del sitio quirúrgico. Microambiente celular, vascularización del lecho quirúrgico, integridad de los colgajos, médula / disponibilidad de células, integridad del coágulo medular, carga cíclica /compresión axial, estabilidad, tamaño del defecto, destrucción de los tejidos blandos e infección local.

- Técnica quirúrgica. Preparación del lecho para injerto, control local de la infección, colocación correcta, estabilidad del injerto y carga mecánica.

- Variables del paciente. Edad, estado nutricional, antecedentes sistémicos, factores de riesgo fumador, enfermedad periodontal, afección de glándulas salivares, terapias, radiación, medicación, características oclusales, respirador oral $\mathrm{y}$ antecedentes psicológicos.

- Injerto. Biocompatibilidad, tamaño de poro adecuado, superficie de contacto con el lecho quirúrgico, volumen adecuado, interfase injerto huésped, capacidad osteoconductiva, capacidad osteoinductiva, presencia de células viables y facilidad de uso. 


\section{Referencias}

1. Vroom MG, Sipos P. Effect of surface topography of screwshaped titanium implants in humans on clinical and radiographic parameters: a 12-year prospective study. Clin Oral Implants Res. 2009; 20(11):1231.

2. Sul YT. Electrochemical growth behavior, surface properties, and enhanced in vivo bone response of $\mathrm{TiO} 2$ nanotubes on microstructured surfaces of blasted, screw-shaped titanium implants. Int J Nanomedicine. 2010; 15(5):87-100.

3. Esposito M, Grusovin MG, Worthington HV. Interventions for replacing missing teeth: treatment of peri-implantitis. Cochrane Database Syst Rev. 2012; 18(5).

4. Palomares KT, Gleason RE, Mason ZD, Cullinane DM, Einhorn TA, Gerstenfeld LC, Morgan EF. Mechanical stimulation alters tissue differentiation and molecular expression during bone healing. J Orthop Res. 2009;27(9):1123-32.

5. Lars L Schropp, Lambros L Kostopoulos, and Ann A Wenzel. Bone healing following immediate versus delayed placement of titanium implants into extraction sockets: a prospective clinical study. J Oral Maxillofac Implants.2003;18(2):189-99.

6. Soardi CM, Bianchi AE, Zandanel E, Spinato S. Clinical and radiographic evaluation of immediately loaded one-piece implants placed into fresh extraction sockets. Quintessence Int. 2012; 43(6):449-56.

7. Vandamme K, Naert I, Geris L, Sloten JV, Puers R, Duyck J. Histodynamics of bone tissue formation around immediately loaded cylindrical implants in the rabbit. Clin Oral Implants Res. 2007; 18(4):471-80.

8. Wennström JL, Derks J. Is there a need for keratinized mucosa around implants to maintain health and tissue stability. Clin Oral Implants Res. 2012; 23 (6):136-46.

9. Chang M, Wennström JL. Soft tissue topography and dimensions lateral to single implant-supported restorations. A crosssectional study. Clin Oral Implants Res. 2013;24(5):556-62.
10. Chang M, Wennström JL. Peri-implant soft tissue and bone crest alterations at fixed dental prostheses: a 3-year prospective study. Clin Oral Implants Res. 2010; 21(5):527-34.

11. Chang M, Wennström JL. Bone alterations at implant-supported FDPs in relation to inter-unit distances: a 5-year radiographic study. Clin Oral Implants Res. 2010;21(7):735-40.

12. Chang M, Wennström JL. Longitudinal changes in tooth/ single-implant relationship and bone topography: an 8-year retrospective analysis. Clin Implant Dent Relat Res. 2012;14(3):388-94.

13. Esposito M, Grusovin MG, Pellegrino G, Soardi E, Felice P. Safety and effectiveness of maxillary early loaded titanium implants with a novel nanostructured calciumincorporated surface (Xpeed): 1-year results from a pilot multicenter randomised controlled trial. J Oral Implantol. 2012;5(3):241-9.

14. Esposito M, Maghaireh H, Grusovin MG, Ziounas I, Worthington HV. Soft tissue management for dental implants: what are the most effective techniques? A Cochrane systematic review. J Oral Implantol. 2012;5(3):221-38.

15. Arismendi JA,Castaño AC,Mejia RM,Mesa AL, Castañeda DA, Tobon SI. Evidencia de cambios clínicos y radiográficos en implantes oseointegrados de superficie maquinada y modificada , 3 y 6 meses de seguimiento.Rev Fac Odontol Univ Antioq. 2006;18(1):6-16.

16. Arismendi JA,Mesa AL,Garcia LP,Salgado JF,Castaño C, Mejia R. Estudio comparativo de implantes de superficie lisa y rugosa .Resultados a 36 meses. Rev Fac Odontol Univ Antioq 2010; 21(2):159-169.

17. Bressan E. Nanostructured Surfaces of Dental Implants. J. Mol. Sci. 2013; 14: 1918-1931. 